\title{
Supporting Information: Tunable and Reversible Substrate Stiffness Reveals Dynamic Mechanosensitivity of Cardiomyocytes
}

\author{
Elise A. Corbin ${ }^{1,2,3, \dagger}$, Alexia Vite ${ }^{3, \dagger}$, Eliot G. Peyster ${ }^{3}$, Myan Bhoopalam4, Jeffrey Brandimarto ${ }^{3}$, \\ Xiao Wang ${ }^{3}$, Alexander I. Bennett ${ }^{5}$, Andy T. Clark ${ }^{6}$, Xuemei Cheng ${ }^{6}$, Kevin T. Turner ${ }^{5}$, Kiran \\ Musunuru $^{3}$, and Kenneth B. Margulies ${ }^{3, *}$ \\ 1 Department of Biomedical Engineering, University of Delaware, Newark, DE, 19716 \\ 2 Department of Materials Science and Engineering, University of Delaware, Newark, DE, 19716 \\ ${ }_{3}^{3}$ Department of Medicine, Division of Cardiology, Perelman School of Medicine, University of \\ Pennsylvania, Philadelphia, PA, 19104 \\ 4 School of Arts and Sciences, University of Pennsylvania, Philadelphia, PA, 19104 \\ ${ }_{5}^{5}$ Department of Mechanical Engineering and Applied Mechanics, School of Engineering and Applied \\ Sciences, University of Pennsylvania, Philadelphia, PA, 19104 \\ ${ }^{6}$ Department of Physics, Bryn Mawr College, Bryn Mawr, PA, 19010
}

† E. A. Corbin and A. Vite contributed equally to this work

* Corresponding author email: kenb@pennmedicine.edu 


\section{Finite Element Method Magnetics Simulation}

Finite element method magnetics (FEMM) was used to analyze the uniformity of magnetic field distribution at the surface of the MRE material. We assumed the magnetic properties of the PDMS were close to other materials that do not exhibit high susceptibility to magnetic fields $(\mu r \approx 1)^{1}$. This is a viable assumption as these materials do not alter an induced magnetic field. The magnetic properties of the permanent magnets were modeled as a NdFeB 40 MGOe magnet with a coercivity of $9.79 \times 10^{5} \mathrm{~A} / \mathrm{m}$ and a relative permeability of 1.049 . The magnetic susceptibility of the doped-PDMS was calculated using the Maxwell-Garnett approximation for magnetic susceptibility of a suspension of non-interacting spherical magnetic particles given by²:

$$
\chi_{M R E}=\frac{3 \varphi \chi_{p}}{\left[3+\chi_{p}(1-\varphi)\right]}
$$

Where $\varphi$ is the volume fraction and $\chi_{p}$ is the magnetic susceptibility of the iron particles. The volume fraction was calculated to be $12 \%$, and the magnetic susceptibility of the iron particles at zero magnetic field, $\chi_{p}=6.875 \times 10^{-4} \mathrm{~m}^{3} / \mathrm{kg}$, was provided by the manufacturer.

\section{Magnetic Flux Density Measurements}

The component of the magnetic flux density normal to the sample plane $\left(B_{z}\right)$ was measured by a Lakeshore model 410 Gauss meter equipped with an axial Hall probe which was fixed at the surface of the MRE. Measurements were taken at specified heights and radial distances by jointly moving the MRE and permanent magnet while keeping the probe fixed. The measurements were repeated without the MRE present while maintaining the same probe to magnet distance.

\section{Ncardia Cardiac Fibroblasts}

Frozen vials of 2 million FibroCor. $4 \mathrm{U}^{\mathrm{TM}}$ fibroblasts were obtained directly from the manufacturer (Ncardia, Cologne, Germany). A T-25 flask was coated with $0.1 \%$ gelatin in PBS without $\mathrm{Ca}^{2+} / \mathrm{Mg}^{2+}$. The flask was then incubated for $1 \mathrm{hr}$ at $37^{\circ} \mathrm{C}$. A vial of cells was thawed in a 
$37^{\circ} \mathrm{C}$ water bath and transferred into $10 \mathrm{~mL}$ Cor. $4 \mathrm{U} \circledast$ medium with ciprofloxacin $\mathrm{HCl}$ at $2 \mu \mathrm{g} / \mathrm{mL}$ (referred to as medium in the following), and kept at $37^{\circ} \mathrm{C}$. The cells were then spun down at 200xg for $4 \mathrm{~min}$, the supernatant was removed, and the cells were resuspended in fresh media. The gelatin solution was removed from the flask and the cells were immediately plated in the flask. Cells were cultured at $37^{\circ} \mathrm{C}, 5 \% \mathrm{CO}_{2}$ and $95 \%$ humidity. Twenty-four hours later, the cell media was exchanged. Three days after the thaw, the cells were lifted with $0.05 \%$ Trypsin for 2 min at $37{ }^{\circ} \mathrm{C}$, spun down at $200 \mathrm{xg}$ for $4 \mathrm{~min}$, re-suspended in fresh media, and plated onto the fibronectin coated magnetorheological elastomers at $100 \mathrm{cells} / \mathrm{mm}^{2}$.

\section{Ncardia Cardiomyocytes}

Frozen vials of 4 million Cor. $4 \mathrm{U} \circledast$ cardiomyocytes (28 days old) were obtained directly from the manufacturer (Ncardia, Cologne, Germany). A T-75 flask was coated with fibronectin at a 1:100 ratio in PBS with $\mathrm{Ca}^{2+} / \mathrm{Mg}^{2+}$. The flask was then incubated for $3 \mathrm{hr}$ at $37^{\circ} \mathrm{C}$ or overnight at $4{ }^{\circ} \mathrm{C}$. A vial of cells was thawed in a $37^{\circ} \mathrm{C}$ water bath and transferred into $10 \mathrm{~mL}$ Cor.4Uß medium with ciprofloxacin $\mathrm{HCl}$ at $2 \mu \mathrm{g} / \mathrm{mL}$ (referred to as medium in the following), and kept at 37 ${ }^{\circ} \mathrm{C}$. The fibronectin solution was removed from the flask and the cells were immediately plated in the flask. Cells were cultured at $37^{\circ} \mathrm{C}, 5 \% \mathrm{CO}_{2}$, and $95 \%$ humidity. Twenty-four hours later, the cell media was exchanged. Three days after the thaw, the cells were lifted with Accumax for 8 min at $37{ }^{\circ} \mathrm{C}$, spun down at $180 \mathrm{xg}$ for $4 \mathrm{~min}$, re-suspended in fresh media, and plated onto the fibronectin coated magnetorheological elastomers at 100 cells $/ \mathrm{mm}^{2}$.

\section{Generation of MYH7-GFP Reporter Line and Differentiation into Cardiomyocytes}

We generated the MYH7-GFP reporter line using the DiPS 1016SevA human induced pluripotent stem cell (iPSC) line (obtained from the Harvard Stem Cell Institute iPSC Core Facility) with previously described techniques. 500-bp homology arms flanking the $M Y H 7$ stop codon were amplified from genomic DNA (DiPS 1016SevA). The 3' homology arm was combined with a viral 2A sequence and the Venus fluorescent protein's gene sequence by PCR, and both homology 
arms were subcloned into the PB-MV1Puro-TK vector (Transposagen), which harbors a piggyBac transposon containing a puromycin selection cassette, to make the targeting vector. The guide RNA was designed by manual inspection of the genomic sequences flanking the MYH7 stop codon. The protospacer sequence (5'-CATCTTGATCTGCTCAGCCC-3') was cloned into the Bbsl site of pGuide (Addgene plasmid \#64711) via the oligonucleotide annealing method. Genome editing was performed using pCas9_GFP (Addgene plasmid \#44719). Upon electroporation of the targeting vector and the CRISPR-Cas9 plasmids into DiPS 1016SevA cells and selection with puromycin as previously described ${ }^{3}$, we obtained several puromycin-resistant clones in which the viral 2A and Venus sequences were correctly knocked into the genome in the heterozygous state, in frame with the MYH7 coding sequence and replacing the stop codon. We pooled these clones and introduced piggyBac to remove the transposons in a scarless fashion as previously described ${ }^{3}$, which yielded a large number of clones in which excision was achieved.

Undifferentiated iPSCs were maintained in StemMACS iPS-Brew XF on tissue culture dishes coated with Geltrex LDEV-Free Reduced Growth Factor Basement Membrane Matrix (ThermoFisher Scientific) in DMEM (Gibco). Then, $0.3 \times 10^{6}$ undifferentiated cells were seeded in each well of a 12-well dish (coated with Geltrex) in iPS-Brew containing $4 \mu \mathrm{M}$ ROCK inhibitor Y27632 (Santa Cruz Biotechnology). The next day, the media was changed to iPS-Brew containing 1 MM CHIR99021 (Cayman Chemical). We then incubated cells in RPMI-B27 (RPMI-1640 from Thermo Fisher Scientific; 2\% B-27 Supplement Minus Insulin from Thermo Fisher Scientific) medium supplemented with recombinant human/mouse/rat activin A ( $50 \mathrm{ng} / \mathrm{mL}$; R\&D Systems) for 1 day; with $5 \mathrm{ng} / \mathrm{mL}$ BMP4 (R\&D Systems) and $1 \mu \mathrm{M}$ CHIR99021 for 2 days; and with $1 \mu \mathrm{M}$ XAV 939 (Thermo Fisher Scientific) for another 2 days; followed by 3 days of incubation in RPMIB27 (minus insulin). The medium was changed to RPMI containing B-27 with insulin (Thermo Fisher Scientific) and 1\% penicillin/streptomycin (Invitrogen) and was subsequently replaced every other day. Around day 12 of differentiation, the medium was changed to cardiac selection 
medium consisting of RPMI (glucose-free) supplemented with B-27 with insulin, L-glutamine, and penicillin/streptomycin. After 3 days, the medium was changed back to RPMI containing B27 with insulin as before, and the cells were allowed to recover over the ensuing days. After 30 days of differentiation, iPSC-derived cardiomyocytes were lifted using $0.25 \%$ trypsin and replated onto fibronectin coated MREs or $35 \mathrm{~mm}$ plastic dishes.

\section{Immunofluorescence}

Cardiomyocytes were stained for filamentous actin (f-actin), sarcomeric a-actinin, yesassociated protein (YAP), and 4',6-diamindino-2-phenylindole (DAPI). Cells were rinsed in PBS, fixed in $4 \%(\mathrm{v} / \mathrm{v})$ paraformaldehyde, permeabilized with $0.1 \%(\mathrm{v} / \mathrm{v})$ Triton X-100 (Sigma-Aldrich) for 5-10 min, and free aldehyde groups were blocked with ammonium chloride for $30 \mathrm{~min}$. Cells were first incubated with rabbit YAP (1:200, Cell Signaling Technology) and mouse sarcomeric a-actinin (1:200, Abcam) primary antibodies overnight at $4{ }^{\circ} \mathrm{C}$ and washed with PBS. Cells were incubated with FITC goat anti-mouse IgG and TRITC goat anti-rabbit IgG secondary antibodies (1:400, Invitrogen) and Alexa Fluor-647 phalloidin (1:50, Sigma-Aldrich) for $2 \mathrm{hr}$ in the dark. Next, DAPI ( $1 \mu \mathrm{g} / \mathrm{mL}$ in sterilized de-ionized water, Sigma-Aldrich) was added for 5-10 min in the dark. Cells were imaged with an upright microscope (Nikon Eclipse 80i) with dip-in lenses. Images were processed using ImageJ.

Flow cytometry data acquisition, deposition, and analysis

For fluorescence-activated cell sorting of the MYH7-GFP reporter iPSC-CMs, cells were cultured on the MRE substrates for $48 \mathrm{hr}$. Cells were treated with $0.25 \%$ trypsin EDTA (Gibco) for 5 mins and then quenched with RPMI media supplemented with $20 \%$ FBS. The cells were spun down at $200 \mathrm{xg}$ for 4 mins, re-suspended in FACS buffer. MYH7-GFP cells were analyzed on a FACSCalibur (Becton Dickinson Immunocytometry Systems, CA) flow cytometer, using CellQuest (version 3.3; Becton Dickinson Immunocytometry Systems) acquisition software. The GFP was collected through a 515/30 nm bandpass filter, and list mode data were acquired for 
20,000 cells defined by a dot plot of FSC versus SSC. Automated quantification was completed with FlowJo 7.5 software. To exclude debris, which tend to have lower forward scatter (FSC) and side scatter (SSC) levels, a gate was placed to remove the low levels. To exclude doublets, which had both very low forward scatter (FSC) and low fluorescence (FL1/2-A), asymmetrical gates were fitted with the auto-gating tool. Using the non-GFP control, which was a simultaneous culture of a non-reporter cell line on a tissue culture dish, we set the gate for a single parameter histogram to distinguish auto-fluorescent levels. The reporter cells can then be analyzed for fluorescent levels.

Fibroblast Stress Fiber Intensity Analysis

Whole slide digital images obtained at 10X magnification comprised the initial study dataset of $\mathrm{N}=60$ images. Images were quality checked, and images with significant artifacts, blurring, and clumping were removed resulting in a final dataset of $N=57$ images $(N=22$ images for ramp up experiment, $\mathrm{N}=35$ images for ramp down experiment). After reviewing the intensity histogram of the study images, some contrast variability due to microscope acquisition time and conditions were noted. Contrast stretching was performed to achieve uniform intensities for dark background and bright outliers/speckles within the images. A histogram transformation method for intensity normalization was not used due to concern over permanently altering the image-toimage phalloidin staining intensity relationships that arise from different culture conditions.

Image analysis was performed in CellProfiler for rapid, unbiased, automated quantification. CellProfiler is an open source highly modular image analysis application that allows for the construction of custom-made processing pipelines through a large library of builtin advanced image analysis algorithms ${ }^{4,5}$. As part of the image analysis pipeline for cell area and intensity quantification, bright outliers were first removed from each image via robust background thresholding ${ }^{6}$ and image subtraction 7 . Subsequently, a two-class adaptive Otsu thresholding method was used to identify objects corresponding to fibroblasts within the image (figure S5). 
Large cell clumps and subcellular fragments/artifacts were excluded using a size filter which limited detected objects to a range of 65 to 400 pixels in diameter. The results of automated area and intensity measurements were exported to spreadsheets for further data analysis. Total area per image was normalized by total cell count on a per-image basis to generate a definitive area per cell measurement. To achieve this, the corresponding DAPI stained images for each phalloidin stained image in the dataset was analyzed in CellProfiler. Robust background thresholding was performed with an intensity threshold of 0.1 to create a binary mask with all DAPI stained nuclei in the foreground. The nuclei were then identified as objects for automated counting using a minimum cross entropy method ${ }^{8,9}$.

\section{Cardiomyocyte Sarcomere Maturation Analysis}

Whole slide digital images obtained at 10X magnification comprised the initial study datasets, with $N=168$ images in the mixed fibroblast-cardiomyocyte culture set and $N=482$ for the dynamic platform stiffness monoculture set.

Due to the nature of the culture process and the large field of view incorporate in each digital micrograph, contours within the culture surface will inevitably create areas of poor focus/blurring within each study image. Diligent quality control (QC) of images to ensure that only high quality images with few artifacts and only small areas of blurring is therefore essential to generating reliable and accurate results. An initial manual QC approach of the digital images was performed at an equivalent of 4.3X magnification (fit-to-screen size). The quality and validity of this QC approach was assessed by calculating the log-log slope and the spatial intensity distribution correlation coefficient at a scale of 10 pixels for each image in the dataset. These statistical parameters have been validated in prior image QC workflows and were used in this set to confirm that a reasonably uniform and high quality image set had been created prior to proceeding with the automated image analysis pipeline. Images approved for inclusion in the final study image set by manual QC were significantly better $(p<0.0001)$ by comparison of mean 
composite QC statistic values when compared to the images flagged for exclusion by manual QC (Table S1). After QC and QC validation, a total of $n=280$ images were included in the final image set for the dynamic stiffness experiment and $n=109$ images were included in the fixed stiffness experiment image set.

Automated object identification and segmentation was performed in Ilastik. Ilastik is an open-source 'machine learning' application designed for automated classification and segmentation $^{10}$, and has been used to enable quantitative analysis of digital images arising from complex culture conditions and advanced microscopy methods ${ }^{11,12}$. Ilastik is designed for realtime, interactive pixel and object classification, enabling a streamlined classifier training workflow. For the development of a mature cardiomyocyte classifier, a supervised learning method was employed in which a subset of the study images were partially annotated to serve as a training exemplars. Annotations for pixel classification were performed to identify 3 pixel classes: 'background', 'mature sarcomere', and 'other cell'. In total, 15 digital images from the culture validation image set underwent partial annotation as part of the initial training for the pixel classification. Labeled pixels serve as the inputs for a random forest classifier, which utilized 35 pixel-level feature detection algorithms to analyze color/intensity, texture, orientation, and edge relations at a range of sigmas from 0.3 to 3.5 pixels. By design, the pixel-level prediction maps generated by pixel-classification training were exquisitely sensitive, accurately identifying virtually all sarcomere regions, but also labeling some regions that were felt to represent other cellular material. Utilizing the prediction map results from pixel-classification of the 15 training images, object-level training was performed on the same images to improve the specificity of the final classifier. For this process, an anisotropic Gaussian smoothing parameter of 1.6 at high magnifications and 2.2 at lower magnification was first applied to the images. A threshold for the pixel-level predictions of 0.4 and a minimum size of 200 pixels in area was then applied to the pixel map to generate a preliminary segmentation of objects. A total of 42 object-level feature 
detection algorithms were applied to each image, analyzing both pixel-level prediction features within the objects themselves and within the neighborhood of objects at a neighborhood size of 30 pixels. The end result of object-level training was two classes of objects: confirmed mature sarcomeres and other cells. The pixel classification and object segmentation workflow was then applied to the full study image dataset.

The exported mature sarcomere classifier results were analyzed in CellProfiler for rapid batch quantification. A global robust background exclusion thresholding method was used to generate a binary mask from the llastik object classification output containing just background and mature sarcomere objects in the foreground. The foreground objects underwent several image transformations (smoothing, dilation, and filling convolutions) to better capture adjacent nuclei associated with the identified mature sarcomere patterns for cell-counting. The associated DAPI-stained image for each actinin-stained image analyzed by the sarcomere classifier was also analyzed in Cell Profiler, with cell-counting performed via the minimum cross entropy method described in the stress fiber experiment section above. By image subtraction, a cell count of only those DAPI stained nuclei present in the areas containing mature sarcomere objects was then performed, allowing for the percentage of total cells that were associated with mature sarcomere pattern to be calculated.

Cardiomyocyte Sarcomere Maturation Analysis Validation

For this validation experiment, nine images not used in classifier training were selected from the co-culture experiment image set. Three members of the research team performed manual identification of areas containing mature sarcomere pattern, annotating digital micrographs to label the position of these areas and counting the total number found on each image. The mature sarcomere classifier then performed the same task on these nine images. Intra-rater agreement was assessed by comparing the initial results from one researcher who performed counting at the time of initial image acquisition to the same researcher's counting 
performed during the validation experiment one month later. Human-to-human inter-rater agreement was assessed by comparing human researcher results to one another and to the average of all three human researchers. Classifier-to-human agreement was assessed by comparing classifier results to each individual human result and to the average of the human manual analysis results. Statistically, percent agreement, Kendall's Tau, and intra-class correlation coefficients were calculated to measure agreement.

A sensitivity analysis was also performed based on these nine images to add additional context to the evaluation of mature sarcomere classifier performance. Because the three human readers annotated the digital images to mark the location of organized sarcomere patterns identified, and because the automated classifier readily performs object segmentation as part of normal workflow (i.e. creates a binary image with identified organized sarcomeres and background), direct sensitivity analyses can be performed via overlaying images.

The nine test images contained 622 mature CMs per manual assessment by human rater \#1 performed at the time of image acquisition. A total of three human raters (including rater \#1 one month after image acquisition) performed independent identification of organized sarcomeres, with results summarized in Table S2.

Intra-rater agreement was assessed by having human rater \#1 perform a second round of counting on these images one month after image acquisition (Table S3). The overall percent difference between the counts obtained by rater 1 was $25.6 \%$, with a Kendall's Tau correlation statistic of 0.69 and an intraclass correlation coefficient of agreement of 0.77 . This represents only modest agreement and correlation, and suggests that manual identification of organized sarcomeres and thus mature CMs lacks reliability.

Human-to-human agreement was assessed by comparing the per-image results obtained by the three human raters. Given the similar variability in results between the two reads of rater 1 and between the reads of rater 1 , rater 2 , and rater 3 , a decision was made to pool all 
four manual reads into the overall manual count mean results. For each human rater assessment of the nine images, the average per-image percent difference from the overall manual mean perimage count was calculated (excluding that rater), as well as a Kendall's Tau correlation statistic comparing each rater's per-image count to the overall mean per-image count. Additionally, a matrix of 1:1 Tau statistics for each possible pair of raters was calculated, with the average of each rater's 1:1 Tau statistics included in Table S4.

Table S5 summarizes the classifier correlation and agreement statistics based on the automated count data. Overall, the classifier demonstrates a similar degree of correlation and agreement to the human raters as is seen between the human raters themselves. Additionally, the intra-class correlation agreement statistic among the raters with and without the inclusion of the automated classifier results was 0.73 , further suggesting that the classifier performs similarly to the human raters.

Automated classifier sensitivity analysis was performed by comparing the objectclassification output of the classifier to the nine manually tagged/annotated images created by the human raters at the time of manual image analysis. Additionally, human rater sensitivity analysis was performed by comparing the tagged/annotated images to one another via the same overlay process. The results of this sensitivity testing are summarized in Table S6. Overall, it is evident that the automated classifier has superior sensitivity than any of the human raters, recognizing areas of organized sarcomeres identified by human raters with an average sensitivity of $91.7 \%$ compared to a mean sensitivity of $74.8 \%$ for the human raters. These sensitivity findings are important, confirming that the classifier not only arrives at similar total counts to the human raters, but also that it is seeing the same organized sarcomere pattern as the human raters to arrive at those counts.

As stated previously, manual object identification by human readers has an inherently high positive predictive value (as human opinion is the standard in defining object identity), but 
lacks the comprehensive methodology and subtle feature identification of automated approaches. As a result, one would expect a somewhat lower CM count result from a human reader compared to an automated classifier, which may explain the relatively poor sensitivity of human readers. However, when examining a cohort of human count data from multiple readers, it is reasonable to assume that the highest count for each sample image will continue to represent a high standard of positive predictive value while also representing the 'best' human performance at thorough image examination. In other words, the highest count will still be identifying true-positive objects, but will also be the manual read with the fewest missed objects. As a result, it is informative to compare the highest manual count for each image in this 9 image validation set with the classifier counts. Table $\mathbf{S 7}$ presents the raw count data, while Table $\mathbf{S 8}$ presents the summary correlation and agreement statistics, revealing a very good degree of correlation and agreement (Tau $=0.87$, ICC Agreement $=0.94)$.

\section{Gene Expression Analysis}

RNA was isolated from iPSC-CM cells by using the Qiagen RNeasy Mini kit (Qiagen Inc., Germantown, MD) with on-column DNAse treatment. RNA quantity was measured by Broad Range RNA assay for the Qubit (Invitrogen). RNA was reverse transcribed using the ABI® High Capacity cDNA Reverse Transcription Kit. Specific primers are listed in Table S9. For each RTqPCR, a standard curve for each gene assay was used to account for the difference of PCR efficiencies between each assay reaction. Data was analyzed using the $\Delta \Delta \mathrm{C}_{\mathrm{T}}$ method using GAPDH as a housekeeping gene.

\section{Magnetic Field Strength Determination}

The equations for the field strength of a permanent magnet are not trivial, and the spatial dispersion of iron particles in our composite complicates the matter further. Because of the small iron particle size (3-4 $\mu \mathrm{m}$ diameter spheres), we have chosen to assume that there is very small lateral $(x, y)$ change in the magnetic field incident on a small field of particles (approx. $100 \mu \mathrm{m} x$ 
$100 \mu \mathrm{m})$. A particle field this size is much larger than the cells in this study but much smaller than the size of the magnetic field. Using this assumption, we can estimate the magnetic pull force on the composite based on the pull force of a smaller, characteristic composite sample. To do this, a $4 \mathrm{~mm}$ diameter cylindrical biopsy of the composite was attached to the sensing end of a $10 \mathrm{~g}$ load cell (Cooper Instruments and Systems, Warrenton, VA, USA). While force data was being recorded (Matlab), the magnet was then moved from far out of range until it made contact with the sample. The magnetic pull-force data from this experiment is given in figure $\mathbf{S 1}$. Repeatability and Cycling of MRE Modulus

The elastic modulus was repeatedly measured on the same device to determine the consistency of a single device to return the same modulus (figure S6A). We found that the stiffness is very repeatable, with minimal variation except for the $50 \%$ sample without spacer. The variation in modulus without the spacer (largest magnetic field gradient) is due to indentation position across the sample. We mapped the variations in modulus radially across the device (figure S6B) for both the no spacer magnet condition as well as no magnet condition. There is an increase in modulus radially for the no spacer magnet condition; however, applying a more uniform field would likely reduce this variability across the sample. We also considered the repeatability of the stiffness of a single device when cycled up and down (figure S6C). Repeated cycling resulted in only minimal variability in properties.

\section{MRE Roughness Characterization}

We characterized the roughness of the MRE surface using white light interferometry (Zygo, NewView 6000). The mean surface roughness (Ra) of the MRE surface without a magnetic field applied was calculated over $1 \times 1 \mathrm{~mm}^{2}$ region (figure S7), and the average surface roughness over this area was determined to be $295 \pm 30 \mathrm{~nm}$. 
A

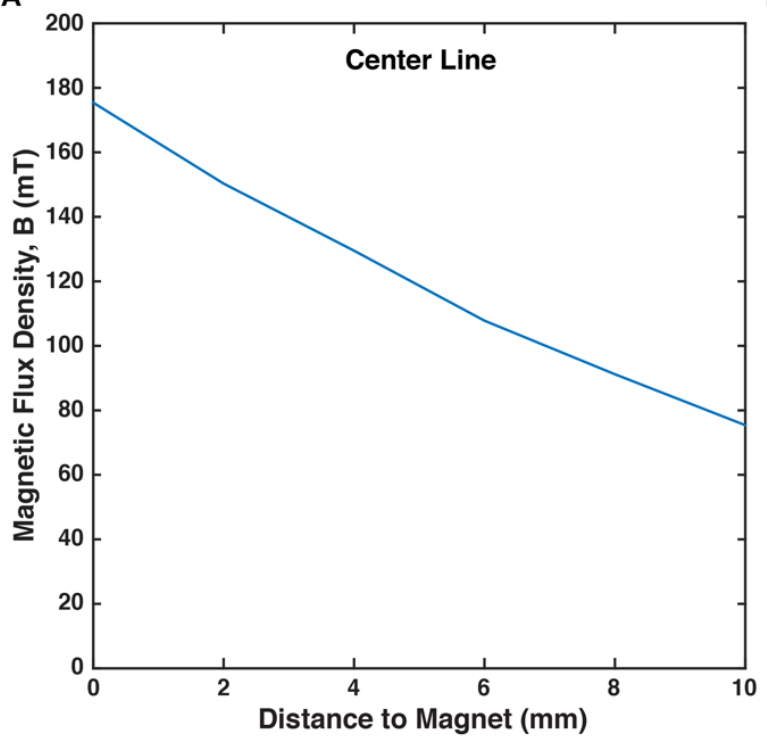

B

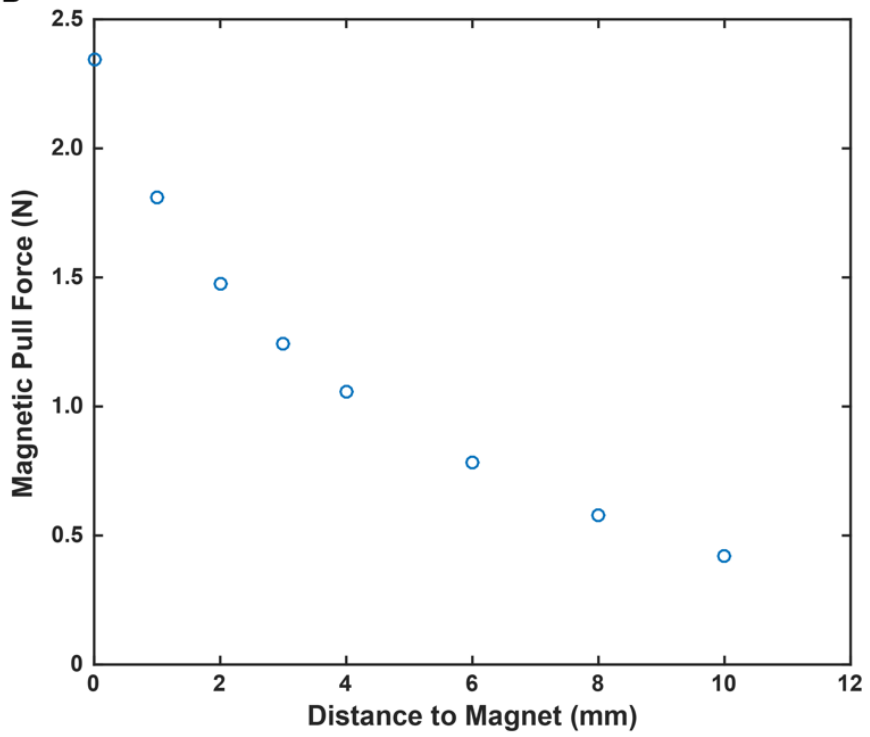

Figure S1. (A) Magnetic Flux Density, B (mT), from the center of the magnet. (B) Magnetic pullforce $(\mathrm{N})$ data as a function of magnet distance $(\mathrm{mm})$.

A

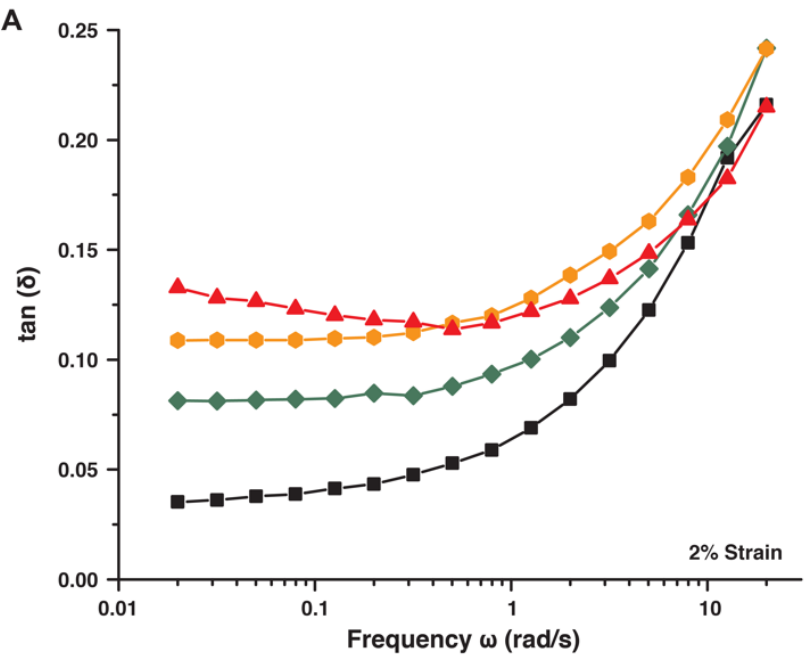

B

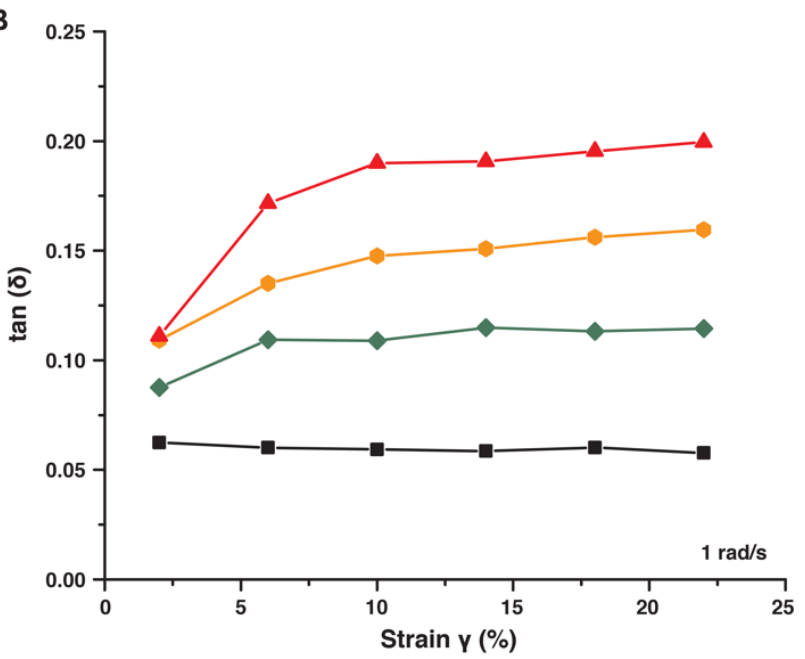

Figure S2. (A-B) Frequency and strain dependence of the loss tangent, a dimensionless ratio of viscoelastic energy loss and energy storage - a measure of the viscoelasticity of soft materials, which is defined as the ratio of the loss modulus (G") to the storage modulus (G') for four magnetic flux densities (from different distances from sample to magnet). 
A

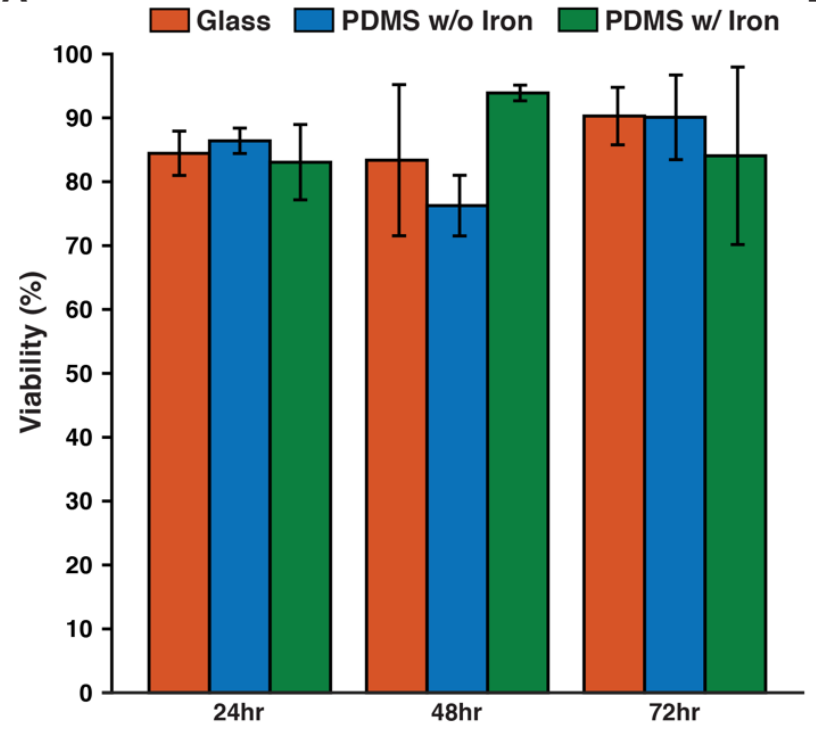

C

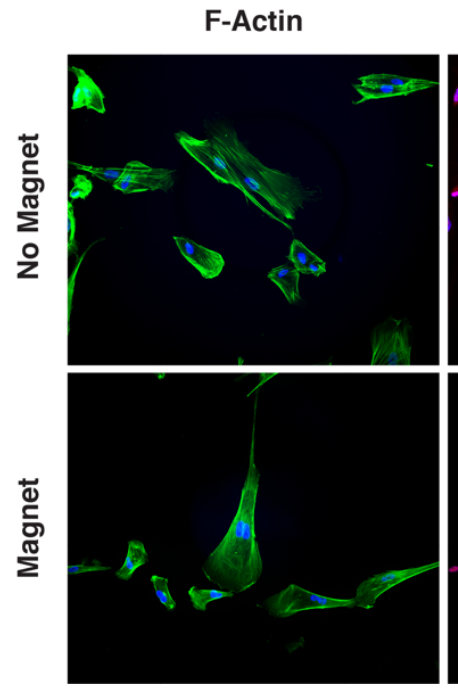

B

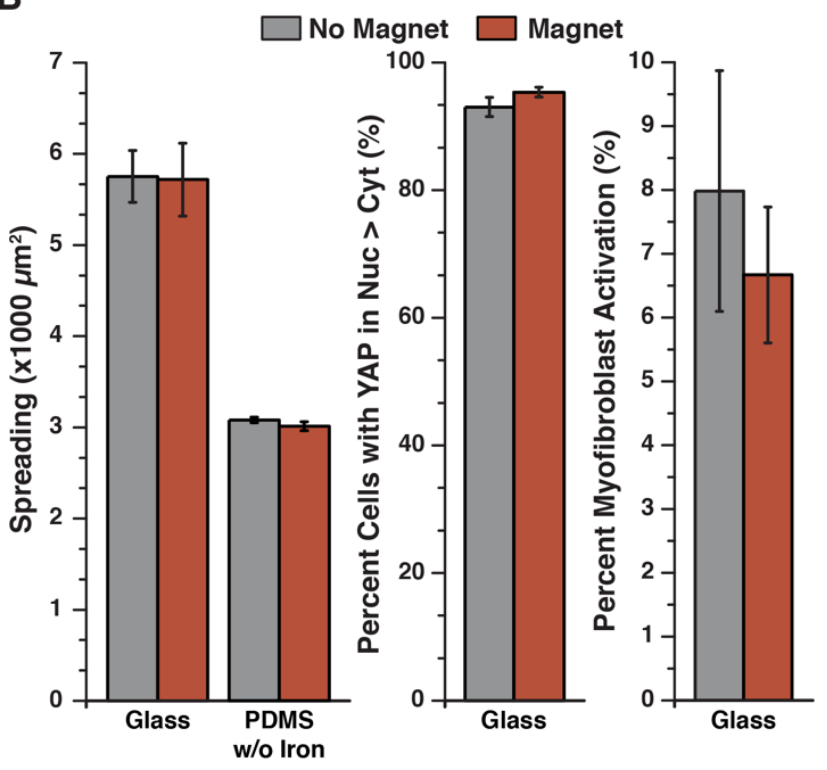

Combined YAP and F-Actin
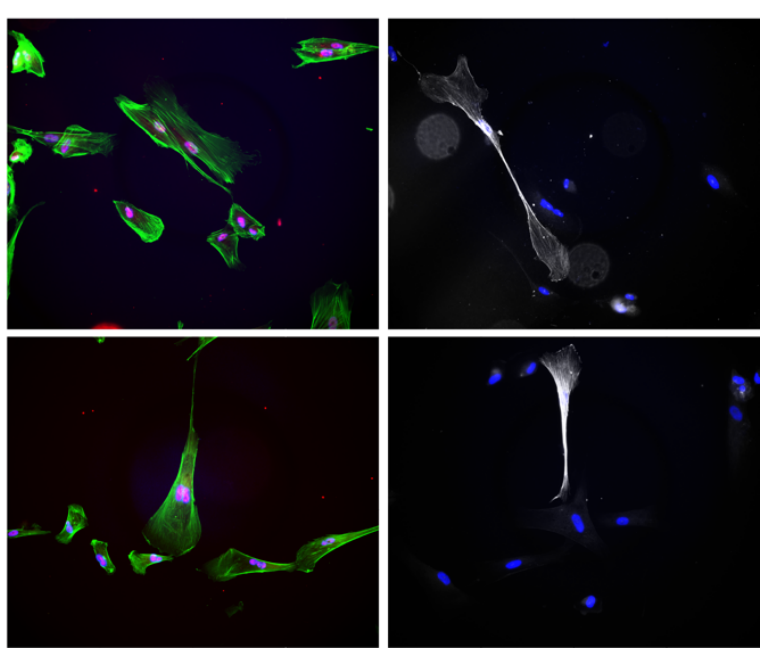

Figure S3. (A) Viability of the neonatal rat fibroblasts over the course of 3 days in culture on a control of glass, PDMS without iron, and PDMS with iron. (B) Magnetic field gradient controls on glass and PDMS without Iron controls comparing the spreading changes, YAP in the nucleus, and myofibroblast activation. (C) Representative images showing the F-Actin, YAP, and alphaSMA in cardiac fibroblasts on glass with and without magnet. 

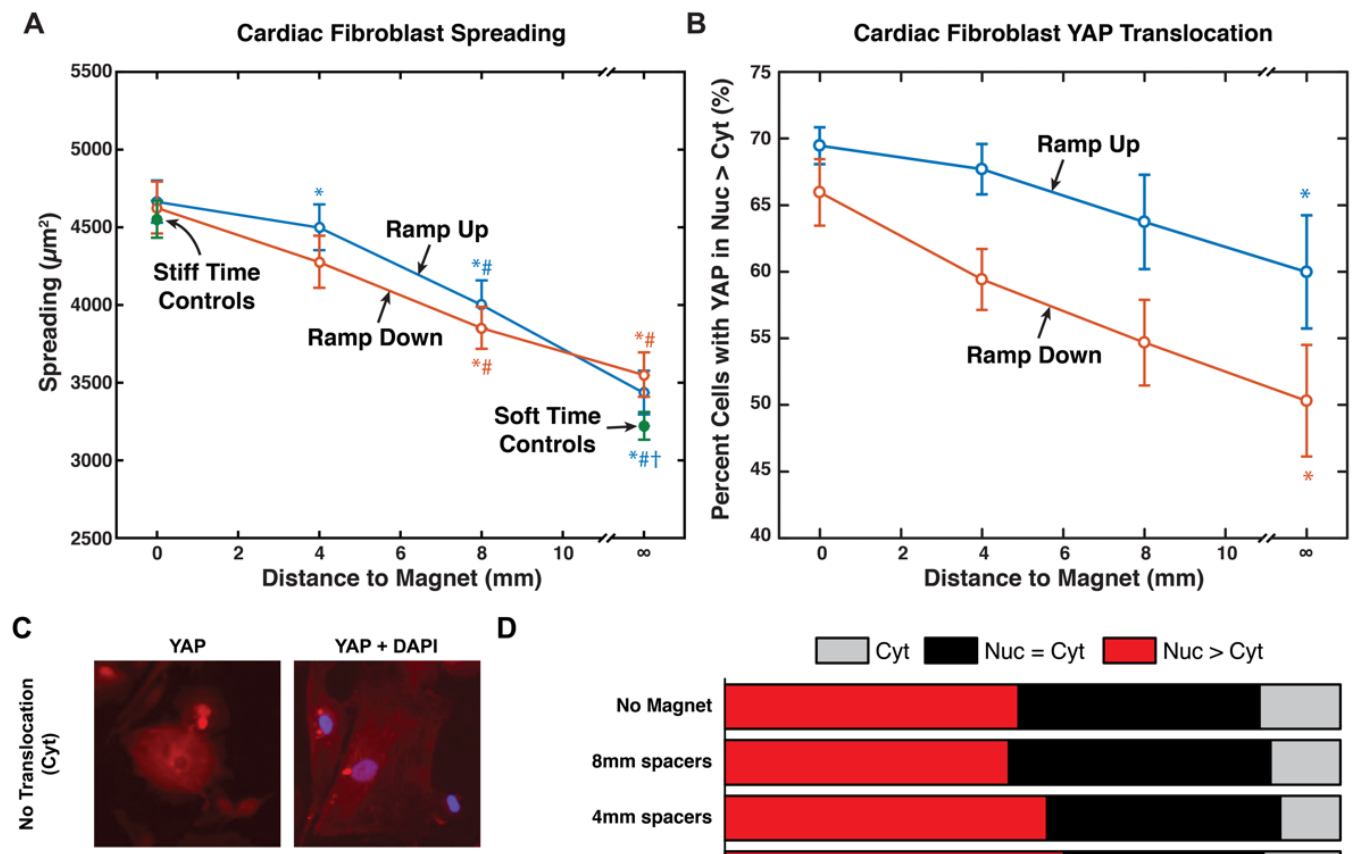

D
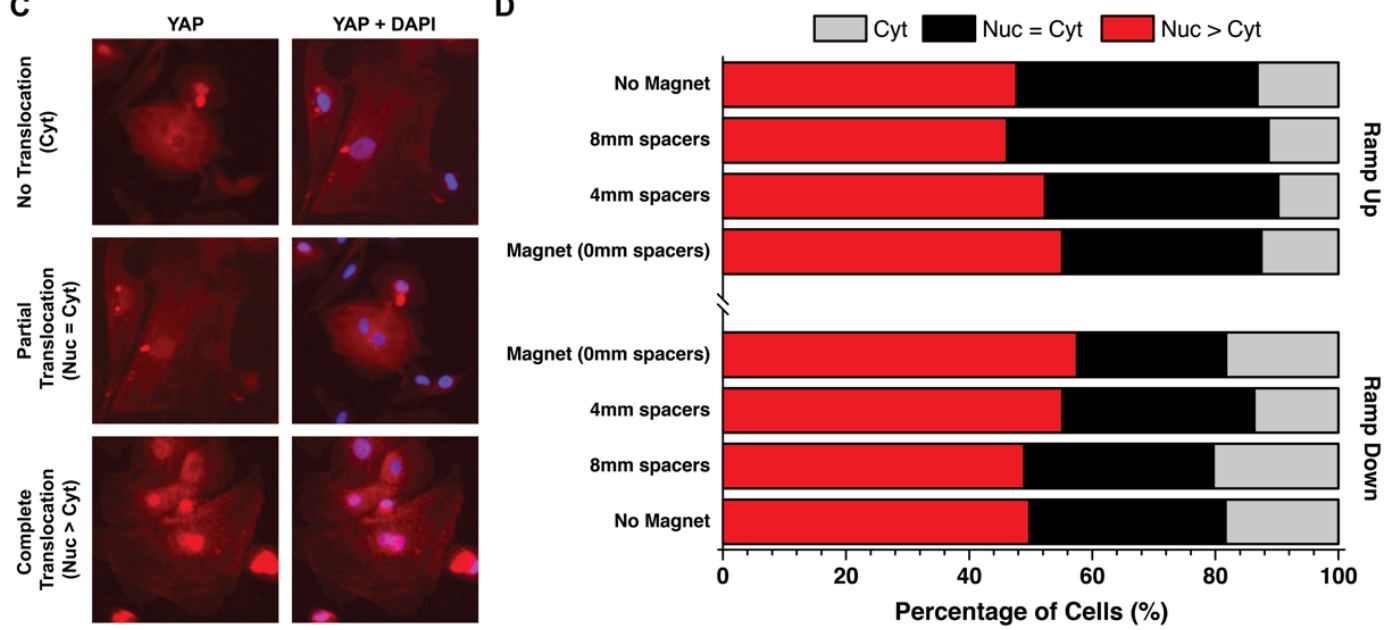

Figure S4. (A) Cardiac fibroblast cell areas were measured to quantify cell spreading as a function of stiffness in both the ramp up (blue) and ramp down (orange). Green dots indicate the positive (stiff) time controls and the negative (soft) time controls. (B) Cardiac fibroblast YAP nuclear translocation was determined as a function of stiffness for both the ramp up and ramp down. (C) Representative images of YAP immunostaining in cardiomyocytes of translocation classifications with the first column showing YAP staining alone and the second column showing YAP counterstained with DAPI. (D) Quantification of YAP translocation with temporal stiffness increases (Ramp Up) and decreases (Ramp Down). Cells were classified into 3 categories based on sub-cellular localization: complete translocation (Nuc > Cyt), partial translocation (Nuc = Cyt), and no translocation (Cyt). 

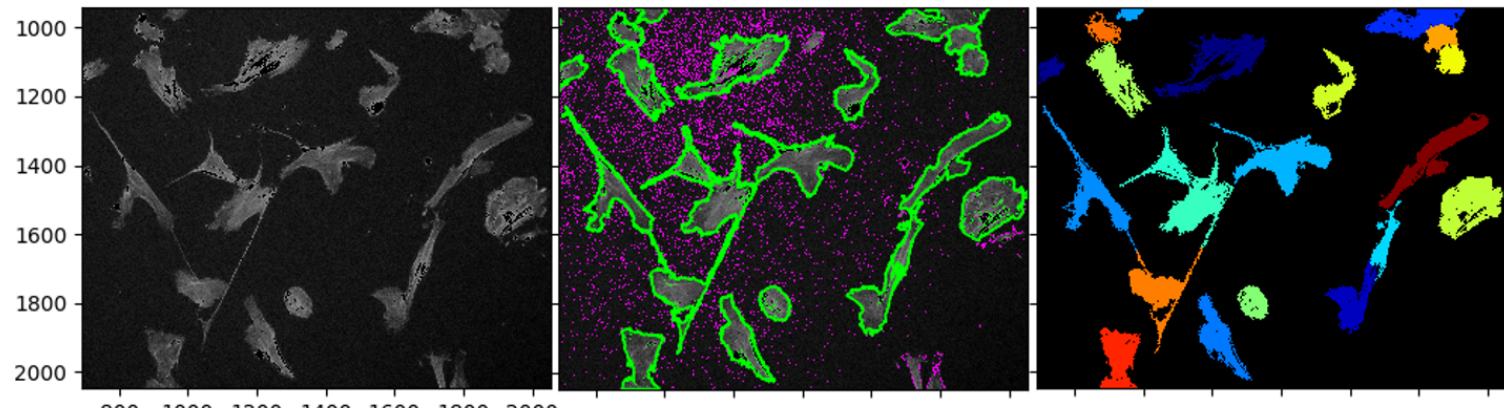

$800 \quad 1000 \quad 1200 \quad 1400 \quad 1600 \quad 1800 \quad 2000$

Figure S5. Sample region for fibroblast size and phalloidin staining intensity automated workflow. Left pane is a region of the raw digital micrograph. Middle pane shows pipelinedesignated fibroblasts for intensity per fibroblast quantification. Right pane shows masked image of pipeline-designated fibroblasts for area quantification.
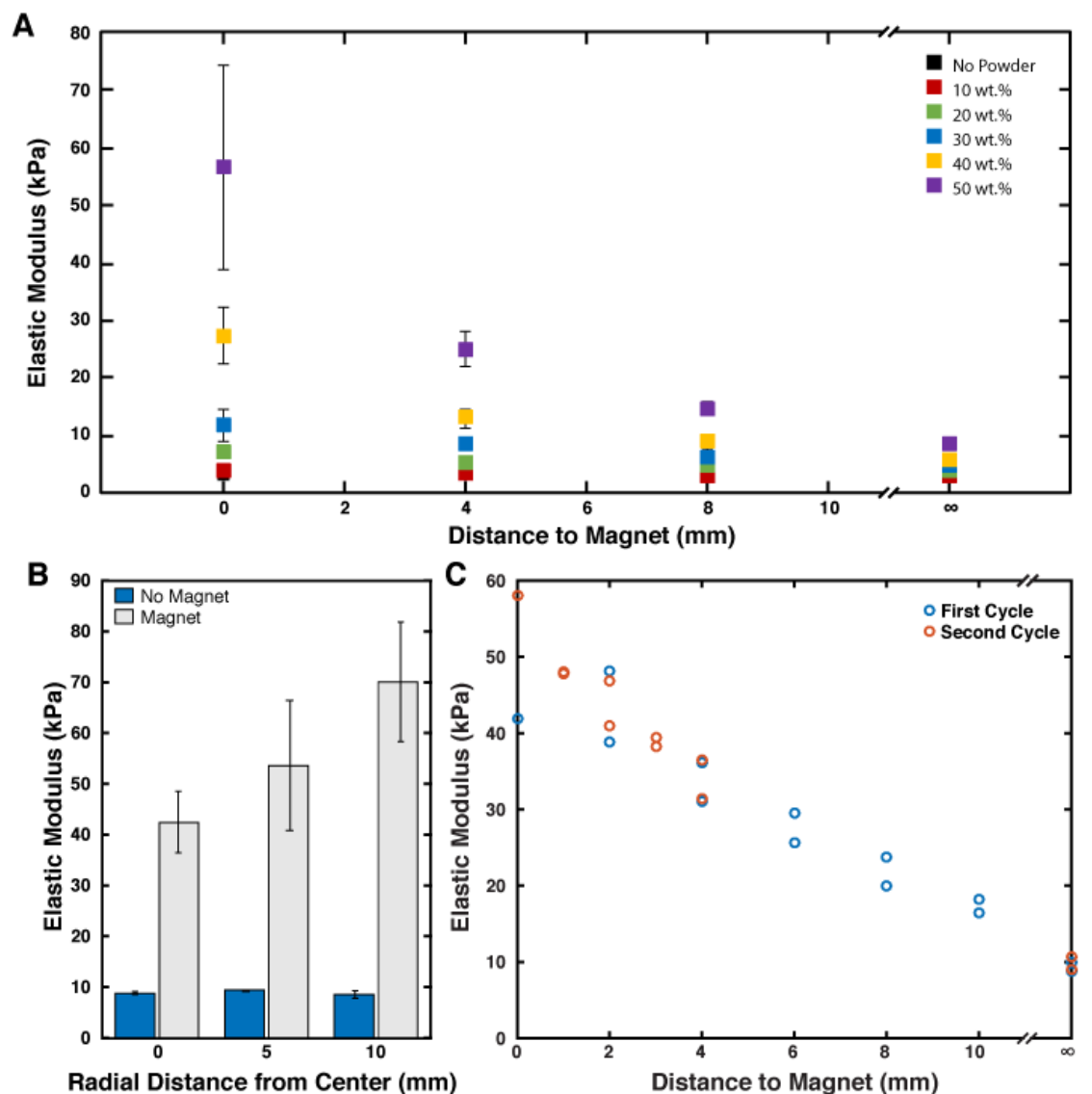

Figure S6. (A) Repeatability of the elastic modulus of single MRE devices with varying wt.\% at set magnet distances. (B) Elastic modulus with and without magnet radially from the center of the device for a device at 50wt.\%. (C) Elastic modulus two cycles of a single device at 50wt.\%. 


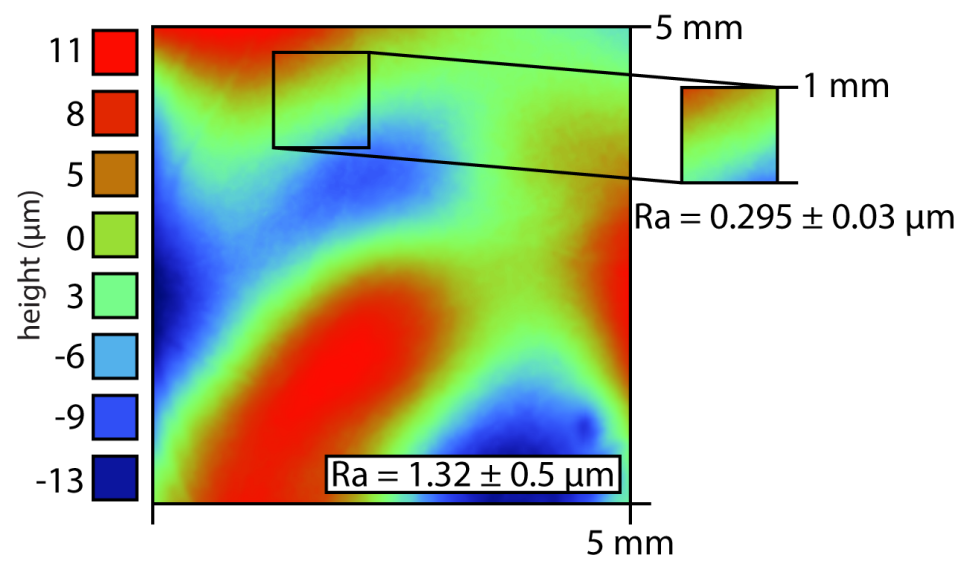

Figure S7. Representative white light interferometer roughness profile of MRE surface without a magnetic field applied. Ra indicates the mean surface roughness calculated over a given region. Ra calculated on a $1 \times 1 \mathrm{~mm}^{2}$ region was $295 \pm 30 \mathrm{~nm}$, and on a $5 \times 5 \mathrm{~mm}^{2}$ region was $1.32 \pm$ $0.5 \mu \mathrm{m}$.

Table S1. Combined QC statistics of the raw actinin stained images for the mature sarcomere automated classifier experiments.

\begin{tabular}{|c|c|c|c|c|c|}
\hline Manual QC & $\begin{array}{c}\text { Number of } \\
\text { Observations }\end{array}$ & $\begin{array}{c}\text { Median } \\
\text { Composite } \\
\text { QC Statistic }\end{array}$ & $\begin{array}{c}95 \% \mathrm{Cl} \text { of } \\
\text { Median }\end{array}$ & $\begin{array}{c}\text { Mean } \\
\text { Composite } \\
\text { QC Statistic }\end{array}$ & SD of Mean \\
\hline Included & 389 & -1.44923 & $\begin{array}{l}-1.477276 \text { to } \\
-1.42069\end{array}$ & -1.45352 & 0.194675 \\
\hline Excluded & 261 & -1.78309 & $\begin{array}{l}-1.843499 \text { to } \\
-1.707777\end{array}$ & -1.81087 & 0.376656 \\
\hline
\end{tabular}

Table S2. Per-Image CM Counts by Human Raters

\begin{tabular}{lccrrrr}
\hline Image & $\begin{array}{c}\text { Rater 1 } \\
\left(\mathbf{1}^{\text {ST }} \text { READ) }\right.\end{array}$ & $\begin{array}{c}\text { Rater 1 } \\
\left(\mathbf{2}^{\text {ND }} \text { READ) }\right.\end{array}$ & Rater 2 & Rater 3 & Per-Image Average & STDev \\
\hline$\# 1$ & 57 & 47 & 40 & 42 & 46.5 & 7.59 \\
$\# 2$ & 76 & 44 & 48 & 25 & 48.25 & 21.05 \\
$\# 3$ & 82 & 67 & 75 & 47 & 67.75 & 15.13
\end{tabular}




\begin{tabular}{lccrccr}
$\# 4$ & 124 & 98 & 100 & 107 & 107.25 & 11.82 \\
$\# 5$ & 28 & 23 & 24 & 20 & 23.75 & 3.3 \\
$\# 6$ & 54 & 34 & 53 & 19 & 40 & 16.75 \\
$\# 7$ & 67 & 75 & 60 & 61 & 65.75 & 6.9 \\
$\# 8$ & 76 & 58 & 60 & 36 & 57.5 & 16.44 \\
$\# 9$ & 58 & 52 & 73 & 40 & 55.75 & 13.72 \\
\hline Total & $\mathbf{6 2 2}$ & $\mathbf{4 9 8}$ & $\mathbf{5 3 3}$ & $\mathbf{3 9 7}$ & $\mathbf{5 1 2 . 5}$ & $\mathbf{9 3 . 0 3}$ \\
\hline
\end{tabular}

Table S3. Intra-rater Agreement

\begin{tabular}{llll}
\hline & \% Difference & Kendall's Tau & $\begin{array}{l}\text { Intra-class Correlation } \\
\text { Coefficient }\end{array}$ \\
\hline Rater 1 & $25.6 \%$ & 0.69 & 0.77 \\
\hline
\end{tabular}

Table S4. Inter-rater Agreement Summary Statistics

\begin{tabular}{lrrr}
\hline & $\begin{array}{l}\text { Average per-image } \\
\text { \% difference from } \\
\text { manual count mean* }\end{array}$ & $\begin{array}{l}\text { Kendall's Tau } \\
\text { (Compared to } \\
\text { manual count mean*) }\end{array}$ & $\begin{array}{l}\text { Average Kendall's } \\
\text { Tau }\end{array}$ \\
\hline Rater 1 (1st Read) & $34.9 \%$ & $0.65(p<0.05)$ & 0.65 \\
Rater 1 (1st Read) & $8.8 \%$ & $0.89(p<0.05)$ & 0.7 \\
Rater 2 & $17.3 \%$ & $0.64(p<0.05)$ & 0.56 \\
Rater 3 & $34.1 \%$ & $0.64(p<0.05)$ & 0.56 \\
\hline Overall & $23.8 \%$ & 0.7 & 0.63 \\
\hline
\end{tabular}

Table S5. Classifier Agreement Summary Statistics

\begin{tabular}{llcr}
\hline & $\begin{array}{l}\text { Average per-image \% } \\
\text { difference from } \\
\text { manual count mean* }\end{array}$ & $\begin{array}{l}\text { Kendall's Tau } \\
\text { (Compared to manual } \\
\text { count mean })\end{array}$ & $\begin{array}{l}\text { Average Kendall's } \\
\text { Tau }^{\S}\end{array}$ \\
\hline Classifier & $30.4 \%$ & $0.78(\mathrm{p}<0.05)$ & 0.63 \\
\hline
\end{tabular}

Table S6. Sensitivity Testing Results

\begin{tabular}{lcccc}
\hline & Rater 1 (2 ${ }^{\text {nd }}$ read) & Rater 2 & Rater 3 & Overall \\
\hline Sensitivity of Classifier & $90.8 \%$ & $91.8 \%$ & $92.4 \%$ & $91.7 \%$ \\
Sensitivity of Human Rater & $82.7 \%$ & $73.6 \%$ & $68.1 \%$ & $74.8 \%$ \\
\hline
\end{tabular}

Table S7. Raw Count Data for Classifier and Highest Manual Count

\begin{tabular}{llllllllll}
\hline Image Number & 1 & 2 & 3 & 4 & 5 & 6 & 7 & 8 & 9 \\
\hline
\end{tabular}




\begin{tabular}{llllllllll}
\hline Classifier Count & 50 & 97 & 87 & 129 & 33 & 40 & 81 & 73 & 71 \\
Highest Manual Count & 57 & 76 & 82 & 124 & 28 & 54 & 75 & 76 & 73 \\
\hline
\end{tabular}

Table S8. Correlation and Agreement Statistics for Classifier-to-Highest Manual Count

Comparison

\begin{tabular}{llll}
\hline & $\begin{array}{l}\text { Avg per-image } \\
\% \text { difference }\end{array}$ & $\begin{array}{l}\text { Kendall's } \\
\text { Tau }\end{array}$ & $\begin{array}{l}\text { Intra-class correlation } \\
\text { coefficient }\end{array}$ \\
\hline $\begin{array}{l}\text { Classifier-to-Highest manual } \\
\text { count comparison }\end{array}$ & $12.0 \%$ & 0.87 & 0.94 \\
\hline
\end{tabular}

Table S9. Primers used for qRT-PCR.

\begin{tabular}{lll}
\hline & Forward & Reverse \\
\hline AURKA & TIT GGG TGG TCA GTA CAT GCT & AGG CTC CAG AGA TCC ACC TT \\
CCNB1 & GTG GAT GCA GAA GAT GGA GC & CCG ACC CAG TAG GTA TIT TGG \\
CTGF & TGT GCA CCG CCA AAG ATG GT & CCA ACC ACG GTT TGG TCC T \\
FN1 & AGC AAG CCC GGT TGT TAT GA & CCC ACT CGG TAA GTG TTC CC \\
GAPDH & GGA AGG TGA AGG TCG GAG TC & GTT GAG GTC AAT GAA GGG GTC \\
MYH6 & CAT CAA CGC AAC CCT GGA & GCT GCT CAA AGC TGT TGA AAT \\
MYH7 & ACT GCC GAG ACC GAG TAT G & GCG ATC CTT GAG GTT GTA GAG C \\
NKX2-5 & GCC GCA CAG TAA TGG TAA GG & CT GAG CCA GCC TGA CTT TC \\
NPPA & GGG TAG GAT TGA CAG GAT TGG & TCG AGC AGA TT GGC TGT TAT \\
NPPB & GCT GCT TTG GGA GGA AGA TG & ATG AGT CAC TTC AAA GGC GG \\
RXRA & GCC AGT ACT GCC GCT ACC A & CGA CTC CAC CTC ATT CTC GT \\
SEMA3A & TाT CAG CAA TGG AGC TाT CC & CAA CAC TCA GCA CAC GCT TT \\
\hline
\end{tabular}




\section{References}

1. Keyes, J., Junkin, M., Wong, P. K. \& Geest, J. P. Vande. Computational Simulation of a Magnetic Microactuator for Tissue Engineering Applications. Biomed Microdevices 11, 1259-1267 (2009).

2. Gorodkin, S. R., James, R. O. \& Kordonski, W. I. Magnetic Properties of Carbonyl Iron Particles in Magnetorheological Fluids. J. Phys. Conf. Ser. 149, 012051 (2009).

3. Pashos, E. E., Park, Y. S., Wang, X., Raghavan, A., Yang, W., Abbey, D., Peters, D. T., Arbelaez, J., Hernandez, M., Kuperwasser, N., Li, W., Lian, Z., Liu, Y., Lv, W., LytleGabbin, S. L., Marchadier, D. H., Rogov, P., Shi, J., Slovik, K. J., Stylianou, I. M., Wang, L., Yan, R., Zhang, X., Kathiresan, S., Duncan, S. A., Mikkelsen, T. S., Morrisey, E. E., Rader, D. J., Brown, C. D. \& Musunuru, K. Large, Diverse Population Cohorts of HiPSCs and Derived Hepatocyte-like Cells Reveal Functional Genetic Variation at Blood LipidAssociated Loci. Cell Stem Cell 20, 558-570.e10 (2017).

4. Carpenter, A. E., Jones, T. R., Lamprecht, M. R., Clarke, C., Kang, I. H., Friman, O., Guertin, D. A., Chang, J. H., Lindquist, R. A., Moffat, J., Golland, P. \& Sabatini, D. M. CellProfiler: Image Analysis Software for Identifying and Quantifying Cell Phenotypes. Genome Biol. 7, (2006).

5. Kamentsky, L., Jones, T. R., Fraser, A., Bray, M. A., Logan, D. J., Madden, K. L., Ljosa, V., Rueden, C., Eliceiri, K. W. \& Carpenter, A. E. Improved Structure, Function and Compatibility for CellProfiler: Modular High-Throughput Image Analysis Software. Bioinformatics 27, 1179-1180 (2011).

6. OTSU, N. A TIreshold Selection Method from Gray-Level Histograms. IEEE Trans. Syst. Man. Cybern. 9, 62-66 (1979).

7. Piccardi, M. Background Subtraction Techniques. IEEE Int. Conf. Syst. Man Cybern. 3099-3104 (2004). doi:10.1116/1.572689

8. Pal, N. R. On Minimum Cross-Entropy Thresholding. Pattern Recognit. 29, 575-580 (1996).

9. $\quad$ Li, C. H. \& Lee, C. K. Minimum Cross Entropy Thresholding. Pattern Recognit. 26, 617625 (1993).

10. Sommer, C., Straehle, C., Ullrich, K. \& Hamprecht, F. A. Ilastik: Interactive Learning and Segmentation Toolkit Heidelberg Collaboratory for Image Processing ( $\mathrm{HCl}$ ), University of Heidelberg. Eighth IEEE Int. Symp. Biomed. Imaging 230-233 (2011).

11. Logan, D. J., Shan, J., Bhatia, S. N. \& Carpenter, A. E. Quantifying Co-Cultured Cell Phenotypes in High-Throughput Using Pixel-Based Classification. Methods 96, 6-11 (2016).

12. Kreshuk, A., Straehle, C. N., Sommer, C., Koethe, U., Cantoni, M., Knott, G. \& Hamprecht, F. A. Automated Detection and Segmentation of Synaptic Contacts in Nearly Isotropic Serial Electron Microscopy Images. PLoS One 6, 1-8 (2011). 